\title{
Exploring finite density QCD phase transition with canonical approach -Power of multiple precision computation-
}

\author{
Shotaro Oka* \\ Institute of Theoretical Physics, Department of Physics, Rikkyo University, Toshima-ku, Tokyo, \\ 171-8501 Japan \\ E-mail: okasho-hato@rikkyo.ac.jp
}

\section{for Zn-Collaboration}

\begin{abstract}
The canonical approach for finite density lattice QCD has a numerical instability. This instability makes it difficult to use the method reliably at the finite real chemical potential region. We studied this instability in detail and found that it is caused by the cancellation of significant digits. In order to reduce the effect of this cancellation, we adopt the multiple precision calculation for our discrete Fourier transformation (DFT) program, and we get the canonical partition function $Z_{\mathrm{C}}(n, T)$ with required accuracy. From the obtained $Z_{\mathrm{C}}(n, T)$, we calculate Lee-Yang zero distribution varying the number of significant digits. As a result, some curves surround the origin in the fugacity plane, but they are moved by varying the number of significant digits. Hence, we conclude that these curves are pseudo phase transition lines, and not real ones.
\end{abstract}

The 33rd International Symposium on Lattice Field Theory

14 -18 July 2015

Kobe International Conference Center, Kobe, Japan*

\footnotetext{
* Speaker.
} 


\section{Introduction}

For a long time, the QCD phase diagram has been studied using a perturbation theory, some models, and heavy ion collider experiments. However, in these methods, we can explore only a limited range of temperature and density. Therefore, we have not exactly known the QCD phase diagram yet.

A numerical simulation of the lattice QCD is a powerful tool to study the QCD phase diagram because this may explore finite temperature and finite density regions. But, in the finite density region, a famous problem - the sign problem- occurs. Hence, we cannot calculate physical quantities in a satisfactory manner.

In this paper, we study the canonical approach[1]. This method may drive away the sign problem; however, a numerical instability alternatively arises in a finite density simulation. We will denote that we can reduce this instability, which is necessary to calculate thermodynamic quantities at the finite real chemical potential (finite density) region.

\section{Sign problem}

In order to calculate thermodynamic quantities, let us consider the grand canonical partition function,

$$
Z_{\mathrm{GC}}(\mu, T)=\int D U\left[\operatorname{det} \Delta\left(x, x^{\prime}, \mu\right)\right]^{N_{f}} e^{-S_{G}},
$$

where $\Delta\left(x, x^{\prime}, \mu\right)$ is the fermion matrix, $S_{G}$ is gauge action, $\mu$ is chemical potential, and $N_{f}$ is the number of flavor. In this study, we use an $O(a)$ improved Wilson fermions 2 and the Iwasaki gauge action[3].

By the definition of Wilson fermions, we can derive $[\operatorname{det} \Delta(\mu)]^{\star}=\operatorname{det} \Delta\left(-\mu^{\star}\right)$. This equation means that, if chemical potential is zero $(\mu=0)$ or pure imaginary $\left(\mu=i \mu_{I}\right)$, $\operatorname{det} \Delta(\mu)$ is real, and otherwise $\operatorname{det} \Delta(\mu)$ is complex. Thus, in a finite density simulation when chemical potential is real, $[\operatorname{det} \Delta(\mu)]^{N_{f}}$ in Eq.2.11 becomes complex, and therefore the standard Monte-Carlo method does not work. This problem is called the sign problem.

\section{Canonical approach and its numerical instability}

It is well known that the grand canonical partition function can be represented with the canonical partition function $Z_{\mathrm{C}}(n, T)$ as the following equation,

$$
Z_{\mathrm{GC}}(\mu, T)=\sum_{n=-\infty}^{\infty} Z_{\mathrm{C}}(n, T)\left(e^{\mu / T}\right)^{n}
$$

where $n$ is net quark number. Then, because $\exp (\mu / T)$ is fugacity, this relation is called the fugacity expansion. We can use the fugacity expansion when we calculate the grand partition function for arbitrary chemical potential. Such a method - to calculate grand canonical quantities from canonical quantities - is called the canonical approach.

The canonical approach can drive the sign problem away from the front. This is because, when we calculate the canonical partition function, we can use the following equation,

$$
Z_{\mathrm{C}}(n, T)=\int_{0}^{2 \pi} d\left(\frac{\mu_{I}}{T}\right) Z_{\mathrm{GC}}\left(\mu=i \mu_{I}, T\right) e^{-i\left(\mu_{I} / T\right) n} .
$$


This equation is just the Fourier transformation of $Z_{\mathrm{GC}}(\mu, T)$ for pure imaginary chemical potential $\mu=i \mu_{I}{ }^{1}$. Note that, in a numerical simulation, we use the discrete Fourier transformation (DFT) as

$$
Z_{\mathrm{C}}(n, T)=\frac{1}{N} \sum_{k=0}^{N-1} Z_{\mathrm{GC}}\left(i \frac{\mu_{I}}{T}=i \frac{2 \pi k}{N}\right) e^{-i(2 \pi k / N) n} .
$$

For pure imaginary chemical potential, the fermion determinant $\operatorname{det} \Delta\left(\mu=i \mu_{I}\right)$ becomes real, and no sign problem occurs if the number of fermions $N_{f}$ is even. Therefore, we may calculate $Z_{\mathrm{C}}(n)$ using Eq. (3.2) without the sign problem.

However, it is known that the canonical approach has a numerical instability [10]. This instability occurs when we perform the discrete Fourier transformation in Eq. 3.3). Fig 1 shows that this instability arises when net baryon number $n_{B}$ becomes large. We can calculate the canonical partition function $Z_{\mathrm{C}}(n)$ with enough accuracy when $n_{B}$ is small (up to $n_{B} \simeq 15$ ), but, for larger $n$, we can not control them well. This phenomenon is called a numerical instability of the canonical approach. Because this instability arises when we calculate $Z_{\mathrm{C}}(n)$, the canonical approach does not work actually.

On the other hand, we can use the reduction formula for the fermion deter-

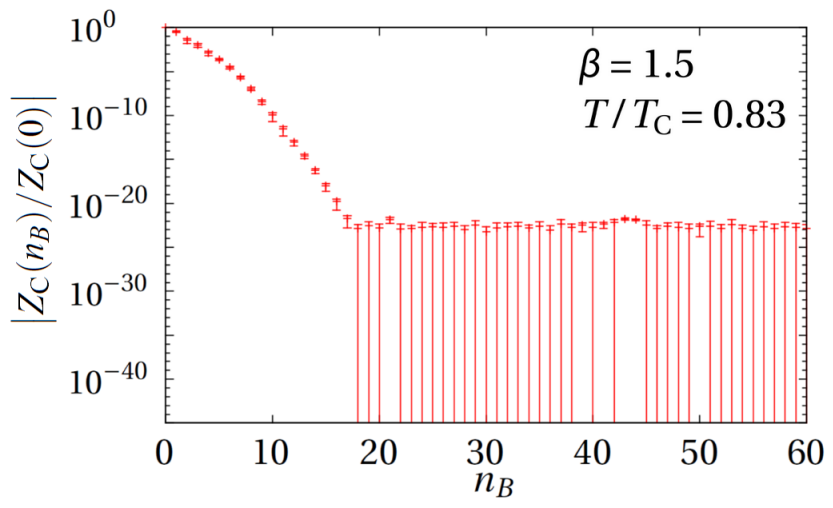

Figure 1: Normalized canonical partition function $\left|Z_{\mathrm{C}}\left(n_{B}\right) / Z_{\mathrm{C}}(0)\right|$ as a function of baryon number $n_{B}$ at $\beta=$ 1.5 (below $T_{c}$ ).

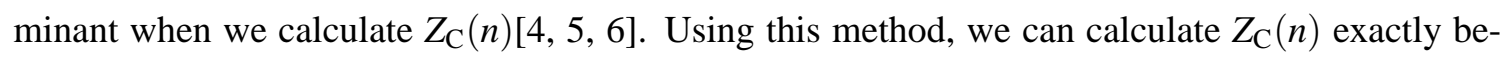
cause it solve a characteristic equation of the determinant and then its eigenvalues relate to $Z_{C}(n)$.

However, this method requires large numerical cost and its result often overflows. The origin of this overflow is that the magnitude of $Z_{C}(n)$ varies drastically when changing $n$. Double precision floating point variables can represent real numbers from $10^{-308}$ to $10^{308}$, but the magnitude of $Z_{C}(n)$ varies beyond this range. We can reduce this overflow by increasing the range of number. Specifically, we can use wide-range numbers [7] or the multiple precision calculation [4] 5]; however, the numerical cost becomes even larger.

In this paper, we will study how to get $Z_{\mathrm{C}}(n)$ with enough accuracy from a different viewpoint.

\section{Solution of numerical instability}

Since Eq. (3.2) can be proved analytically, we can consider that this numerical instability is caused by numerical errors. Numerical errors can be classified into following types of error (see also Refs. [8] and [9]).

(1) Rounding error: for a numerical calculation, numerical values are always rounding when they are assigned to each variable since variables have only the finite precision. Hence, there is a difference between a numerical value and its exact mathematical value; this difference is called the rounding error. For example, this error arises in the following type of calculation:

\footnotetext{
${ }^{1}$ Eqs.3.1 and 3.2 look like tautology, but Eq.3.1 holds for any number $\mu$ and Eq. 3.2 holds only for pure imaginary $\mu=i \mu_{I}$. They are not equivalent at all.
} 


$$
\begin{aligned}
& \frac{1}{3}=3.333333 \cdots \times 10^{-1} \stackrel{\text { assignment to a variable }}{\longrightarrow} 3.333333 \times 10^{-1} . \\
& \text { (Exact mathematical value) } \\
& \text { (Numerical value; } 7 \text { digits) }
\end{aligned}
$$

In above case, the exact mathematical value has infinite significant digits; however the numerical value has only seven significant digits. The numerical result is less than the exact mathematical one as $3.333 \cdots \times 10^{-8}$. Then, to perform rounding exactly, computers usually have a guard digit [9].

(2) Truncation error: when we estimate an infinite series numerically, we must truncate it. Then, there is a difference between the exact mathematical result and its truncated one. This difference is called the truncation error. We can estimate the effect of this error by varying a truncation point of a series.

(3) Cancellation of significant digits: when we calculate a subtraction between two nearly equal values, a lot of higher significant digits are cancelled, therefore the result has only a few significant digits since the number of significant digits is limited for numerical calculation. This phenomenon is called the cancellation of significant digits. Specifically, it occurs the following type of calculation:

$$
\begin{array}{ll}
1.234567-1.234566= & 0.000001 . \\
(7 \text { significant digits }) & (1 \text { significant digit })
\end{array}
$$

In this case, variables have seven significant digits, and six significant digits are lost in this subtraction. If we want to reduce the effect of this cancellation, we should increase the number of significant digits. For instance, we can consider the following calculation instead of above one:

$$
\begin{array}{ll}
1.2345674444444444444444-1.2345661111111111111111= & 0.0000013333333333333333 . \\
(22 \text { significant digits }) & (16 \text { significant digits })
\end{array}
$$

Six significant digits are similarly lost in this calculation, and yet 16 significant digits still remain in the final result.

(4) Loss of trailing digits: when we calculate an addition or subtraction between a huge number and small one, many lower significant digits of the small number are cut since variables hold only the finite precision. Then, the numerical result is underestimated than the mathematical result. This phenomenon is the loss of trailing digits. Specifically, it arises the following type of calculation:

$$
3 \times 10^{10}+2 \times 10^{0}=3.000000002 \times 10^{10} \stackrel{\text { assignment }}{\longrightarrow} 3.000000 \times 10^{10} .
$$

(Exact mathematical result) (Numerical result; 7 digits)

In this case, variables have seven significant digits, and the numerical result is underestimated than the mathematical one as $2 \times 10^{-1}$. As the cancellation of significant digits, we should increase the number of significant digits if we want to reduce this loss.

$$
\begin{aligned}
3 \times 10^{10}+2 \times 10^{0}= & 3.000000002 \times 10^{10} \stackrel{\text { assignment }}{\longrightarrow} 3.000000002000000 \times 10^{10} . \\
& (\text { Exact mathematical result) } \quad \text { (Numerical result; } 16 \text { digits })
\end{aligned}
$$

In this calculation, by increasing significant digits, we can reduce an error between the mathematical result and numerical one. 
In order to make the method to be reliable and promising tool, it is important to realize what occurs in the canonical approach.

Let us consider each type of error. Among these, we can neglect the rounding and the truncation error because of following reasons.

(1) Usually, the rounding error is not transmitted to higher significant digits since computers have a guard digit ${ }^{2}$. If this error seems to be transmitted to higher significant digits, then the cancellation of significant digits or the loss of trailing digits take place. In this paper, we consider that the transmission of rounding error is just an effect of the cancellation and loss.

(2) We do not truncate Fourier series in Eq. 3.3. However, if we use the hopping parameter expansion or the winding number expansion when we calculate the fermion determinant $\Delta(\mu)$, we should truncate an infinite series of these expansion. Then, a truncation error arises, but we can control it by changing a truncation point of this series.

Therefore, this numerical instability is caused by the cancellation of significant digits, the loss of trailing digits, or both.

In this work, we actually monitored the behavior of all variables in our DFT program to study the effect of these two errors. As a result, we found that this numerical instability is caused by the cancellation of significant digits. This cancellation arises mainly at the last addition of the summation in Eq.3.3); specifically,

$$
Z_{\mathrm{C}}(n, T)=\frac{1}{N}\left[\left(\sum_{k=0}^{N-2} Z_{\mathrm{GC}}\left(\frac{2 \pi k}{N}\right) e^{-i(2 \pi k / N) n}\right)+Z_{\mathrm{GC}}\left(\frac{2 \pi(N-1)}{N}\right) e^{-i(2 \pi(N-1) / N) n}\right]
$$

$\uparrow$ This addition.

Fig 2 shows how many digits are lost at this addition in Eq.(4.6). For example, at $n_{B}=50$, about 90 significant digits are lost below $T_{c}$ and about 50 digits are lost above $T_{c}$. From this figure, we can see that, in both temperature, this cancellation becomes serious when $n_{B}$ becomes large.

As explained above, the cancellation of significant digits can be reduced by increasing the number of significant digits. Fig 3 shows the relation between a result of $Z_{\mathrm{C}}(n)$ and the number of sig-

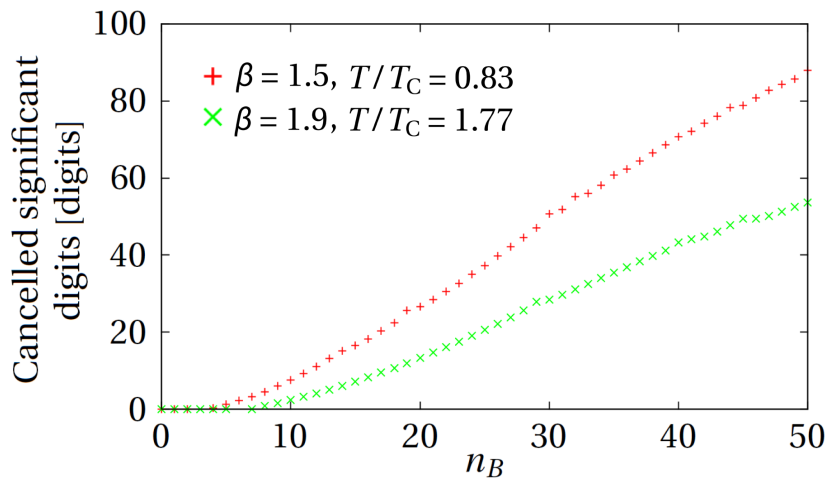

Figure 2: Cancelled significant digits of DFT program as a function of net baryon number $n_{B}$. Upper red points are data below $T_{c}$ and lower green points are data above $T_{c}$. nificant digits. From this figure, we can see that, by increasing the number of significant digits, we can get $Z_{\mathrm{C}}(n)$ very reliably in both temperature.

$Z_{\mathrm{C}}(n)$ becomes small rapidly when increasing net quark number $n$. Then, the cancellation of significant digits becomes severe. Since $Z_{\mathrm{C}}(n)$ is just a residue of this cancellation, the number of cancelled significant digits in Fig, directly relates to a magnitude of $Z_{\mathrm{C}}(n)$ in Fig 3

\footnotetext{
${ }^{2}$ See Ref.9 for the guard bit; it is a detailed review for modern floating point arithmetic.
} 


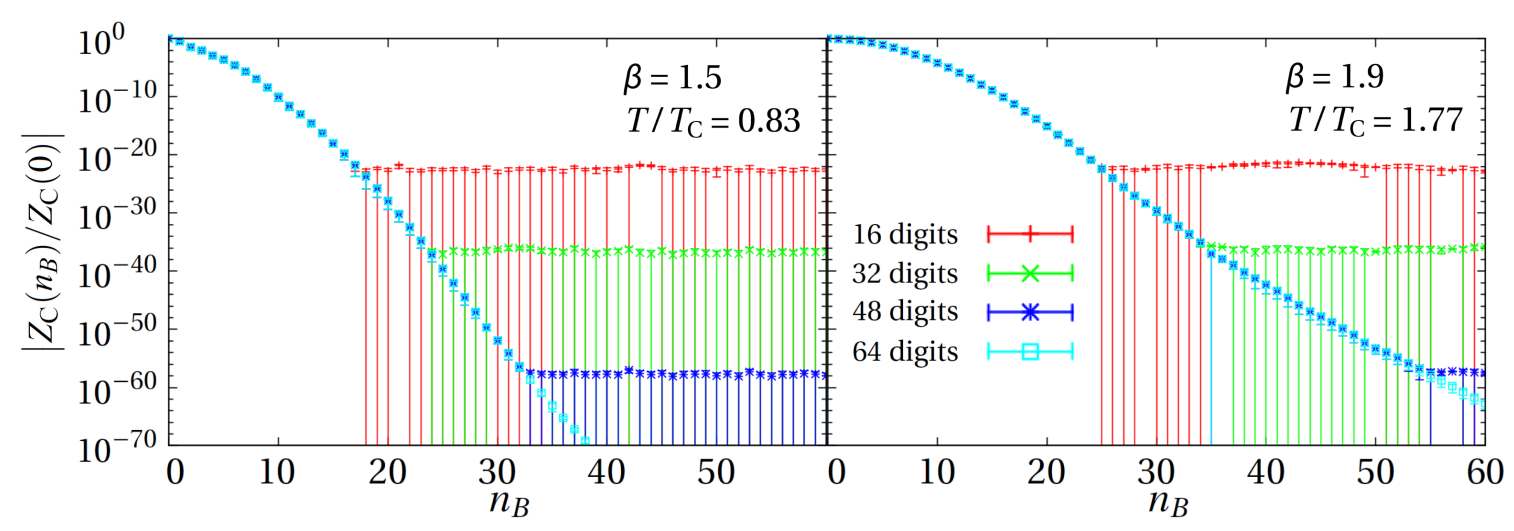

Figure 3: Normalized canonical partition function as a function of $n_{B}$ and the number of significant digits. Left panel shows data below $T_{c}$ and right panel shows data above $T_{c}$. In both temperature, red, green, blue, and cyan points are calculated with 16, 32, 48, and 64 significant digits, respectively.

When we change the lattice volume $V$ with fixing the number of significant digits, a saturation point (kink) of $Z_{\mathrm{C}}(n)$ moves. This is because the net quark number density $n / V$ varies when we change $V$ and fix $n$. When $n / V$ is increased and temperature $T$ is fixed, $Z_{\mathrm{C}}(n, V, T)$ becomes small since it then corresponds to an occurrence probability of excited states. If we increase $n$ and fix $V$ and $T, Z_{\mathrm{C}}(n, V, T)$ becomes small as Fig 3. However, if we increase $V$ and fix $n$ and $T, Z_{\mathrm{C}}(n, V, T)$ becomes large. This means that a kink of $Z_{\mathrm{C}}(n, V, T)$ moves toward larger $n$ region when we increase lattice volume $V$.

\section{Lee-Yang zero distribution using multiple precision canonical approach}

\subsection{Our numerical setup}

We use the Iwasaki gauge action[3] and a clover improved Wilson fermions [2]. When we calculate $\operatorname{det} \Delta(\mu)$ at pure

\begin{tabular}{cccccc}
$\beta$ & $C_{\mathrm{SW}}$ & $\kappa$ & $T / T_{c}$ & $m_{\pi} / m_{\rho}$ & \#Conf. \\
\hline 1.5 & 1.1 & 0.136 & 0.83 & $0.756(13)$ & 100 \\
1.9 & 1.1 & 0.125 & 1.77 & $0.714(15)$ & 600
\end{tabular}

Table 1: Parameters of each simulation. imaginary chemical potential, we use the winding parameter expansion [10] and a reweighting technique (see Ref.[11]). The number of flavor is set to $N_{f}=2$.

We use the APE stout smeared gauge link[12]. Tab 1 shows parameters of each simulation. The multiple precision calculation is applied only in our DFT program and Lee-Yang zero exploring program, where the number of significant digits is set to 400. In other words, we make gauge configurations and calculate $W_{m}$ with double precision variables (16 significant digits). In our DFT program, the division number of an integration interval is $N=512$.

\subsection{Results of Lee-Yang zero distribution}

Lee-Yang zeros (LYZ)[13] are zeros of the grand partition function on the complex chemical potential or fugacity plane. These points represent phase transition points, and we can thus extract some informations of phase transition from their distribution.

Fig, 4 shows LYZ distribution below $T_{c}$ and above $T_{c}$ (lattice size: $8^{3} \times 4$ ). Above $T_{c}$, three straight lines extend radially to origin. Cross points of these lines and the unit circle correspond to the Roberge-Weiss phase transition[14] that is a characteristic phase transition in the deconfinement phase at pure imaginary chemical potential. In both temperature, there are some curves which are surrounding the origin. We might usually think that these curves are phase transition lines and 

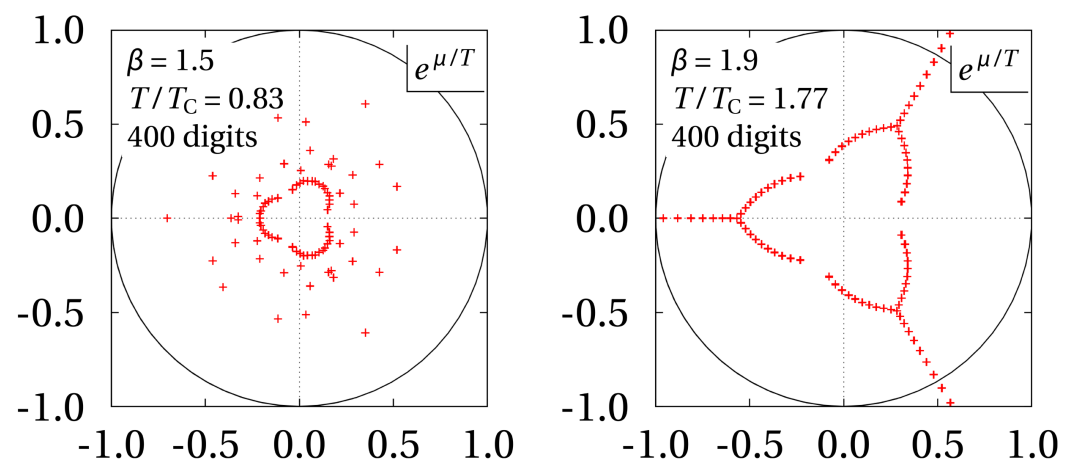

Figure 4: $\mathrm{LYZ}$ distribution of $8^{3} \times 4$ lattice on the fugacity $\exp (\mu / T)$ plane. Left panel shows data below $T_{c}$ and right panel shows data above $T_{c}$. Here, black circles are just unit circles.

the inside and outside of these curves are different phase. However, as described below, it is an artifact.
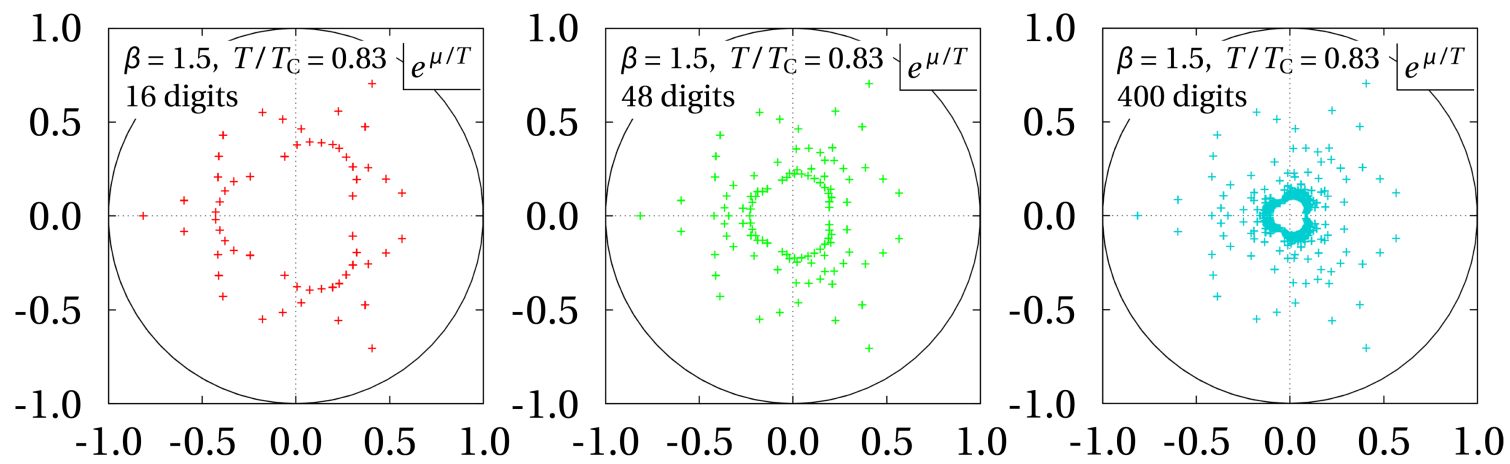

Figure 5: LYZ distribution of $12^{3} \times 4$ lattice below $T_{c}$ on the fugacity $\exp (\mu / T)$ plane. Red, green, and cyan points are calculated with 16,48 , and 400 significant digits, respectively. Here, black circles are just unit circles.

Fig 5 shows LYZ distribution below $T_{c}$ when varying the number of significant digits ${ }^{3}$ (lattice size: $12^{3} \times 4$; this is a preliminary result). Such curves are moving when we vary the number of significant digits. Thus, we conclude that these curves are pseudo phase transition lines, and not real ones.

\section{Acknowledgments}

This study was conducted for $\mathrm{Zn}-$ Collaboration. The author is grateful to members of $\mathrm{Zn}-$ Collaboration, R. Fukuda, A. Nakamura, S. Sakai, A. Suzuki, and Y. Taniguchi for their powerful support. The author thanks Prof. T. Eguchi for his valuable discussion and encouragement. The author appreciates very useful comments by Prof. Ph. de Forcrand and Prof. A. Alexandru. This work is in part based on Lattice QCD common code Bridge++. These calculations were performed using SX-9 and SX-ACE at Research Center for Nuclear Physics and SR16000 at Yukawa Institute for Theoretical Physics. This work is partially supported by Kakenhi $15 \mathrm{H} 03663$ and 26610072.

${ }^{3}$ Then, we check $Z_{\mathrm{C}}(n)$ results which are calculated with 16,48 , and 400 significant digits, and if the saturation (kink) of $Z_{\mathrm{C}}(n)$ appears, we then truncate each $Z_{\mathrm{C}}(n)$ at this point, respectively. After these processing, we calculate Lee-Yang zeros using Eq. 3.1] with 16, 48, and 400 digits. 


\section{References}

[1] A. Hasenfratz and D. Toussaint, Nucl. Phys. B 371 (1992) 539.

[2] B. Sheikholeslami and R. Wohlert, Nucl. Phys. B 259 (1985) 572.

[3] Y. Iwasaki, Nucl. Phys. B 258 (1985) 141.

[4] Ph. de Forcrand and S. Kratochvila, Nucl. Phys. B (Proc. Suppl.) 153 (2006) 62

[5] A. Li, A. Alexandru, and K-F. Liu, Phys.Rev. D 84 (2011) 071503.

[6] K. Nagata and A. Nakamura, Phys. Rev. D 82 (2010) 094027.

[7] K. Nagata, S. Motoki, Y. Nakagawa, A. Nakamura, and T. Saito, Prog. Theor. Exp. Phys. (2012) 01A103.

[8] D. E. Knuth, The Art of Computer Programming, Volume 2 / Seminumerical Algorithms, Second Edition, Addison-Wesley publishing (1969).

[9] D. Goldberg, ACM Computing Surveys (CSUR) 23.1 (1991) 5.

[10] A. Alexandru, M. Faber, I. Horvath, and K-F. Liu, Phys. Rev. D 72 (2010) 114513.

[11] R. Fukuda, A. Nakamura, S. Oka, S. Sakai, A. Suzuki, and Y. Taniguchi, in proceedings of LATTICE2015 (2015).

[12] C. Morningstar and M. J. Peardon, Phys. Rev. D 69 (2004) 054501.

[13] C. N. Yang and T. D. Lee, Phys. Rev. 87 (1952) 404.

[14] A. Roberge and N. Weiss, Nucl. Phys. B 275 (1986) 734. 